\title{
Photoelectric properties of single crystals $\mathrm{Ag}_{3} \mathrm{In}_{5} \mathrm{Se}_{9}$
}

\author{
A.H. Huseynov, R.M. Mamedov \\ Baku State University, Department of Physics, \\ Z. Khalilov str., 23, AZ-1148 Baku, Azerbaijan \\ E-mail: rovshan63@rambler.ru
}

\begin{abstract}
Low-resistance and high-resistance single crystals of $\mathrm{Ag}_{3} \mathrm{In}_{5} \mathrm{Se}_{9}$ compound have been grown using the methods of zone recrystallization and slow cooling at a constant gradient of temperature. We have investigated spectral and lux-ampere characteristics of photoconductivity and determined the mechanism of recombination inherent to non-equilibrium current carriers. It has been ascertained that the capture of electrons emitted by donor centers is caused by a strong electric field applied to a sample.
\end{abstract}

Keywords: single crystal, $\mathrm{Ag}_{3} \mathrm{In}_{5} \mathrm{Se}_{9}$, thermostimulated current, photoconductivity, luxampere characteristic, recombination.

Manuscript received 07.02.06; accepted for publication 23.10.06.

\section{Introduction}

In the work [1], authors reported the existence of a new class of ternary semiconductor compounds with the general formula $\mathrm{A}_{3}^{\mathrm{I}} \mathrm{B}_{5}^{\mathrm{III}} \mathrm{C}_{9}^{\mathrm{VI}}$. The further researches $[2,3]$ showed these compounds to be promissing in manufacturing the photoconverters capable to work under conditions of high temperatures as well as increased frequencies of following electric pulses. In this work, we give the results of photoelectric researches in $\mathrm{Ag}_{3} \mathrm{In}_{5} \mathrm{Se}_{9}$.

\section{Results and discussion}

The spectrum of photoconductivity inherent to $\mathrm{Ag}_{3} \mathrm{In}_{5} \mathrm{Se}_{9}$ was investigated in the samples grown by methods of zone crystallization (a series of low-ohmic samples - LO) and slow cooling (a series of high-ohmic samples - HO), at a constant temperature gradient. Conductivity of the samples and the mobility of current carriers in them had the following values at the room temperature:

1) for $\mathrm{HO}-\sigma=4.6 \cdot 10^{-7} \mathrm{Ohm}^{-1} \mathrm{~cm}^{-1}$;

$$
\mu=120 \mathrm{~cm}^{2} /(\mathrm{V} \cdot \mathrm{s}) \text {; }
$$

2) for $\mathrm{LO}-\sigma=3 \cdot 10^{-5} \mathrm{Ohm}^{-1} \mathrm{~cm}^{-1}$;

$$
\mu=40 \mathrm{~cm}^{2} /(\mathrm{V} \cdot \mathrm{s}) \text {. }
$$

Using the X-ray analysis, it was revealed that $\mathrm{Ag}_{3} \mathrm{In}_{5} \mathrm{Se}_{9}$ single crystals have a hexagonal elementary cell with lattice parameters $a=8.01 \AA, c=16.46 \AA$.
Studying the photoconductivity was carried out both in stationary, and in non-stationary modes.

The typical spectra of photoconductivity (normalized per unity quantum flux) LO- also HOsamples are shown in Fig. 1. Curves 1 and 3 were taken at $100 \mathrm{~K}$, curve $2-$ at $300 \mathrm{~K}$. Beside it, curves 1 and 2 represents spectrum in a mode of modulated $(82 \mathrm{~Hz})$ illumination of the sample.

Low-ohmic crystals are photosensitive within the quantum energy range $1 \ldots 1.8 \mathrm{eV}$, and high-ohmic crystals, in comparison with low-ohmic, are photosensitive in a wider area from the side of high energy stimulating quantum. The spectrum of photoconductivity in all these crystals begins with area of impurity absorption that is located at the edge of fundamental absorption $(1 \ldots 1.2 \mathrm{eV})$ and is overlapped with it. At the same time, it is difficult to determine the value of the forbidden band width $\Delta E_{g}$ using the Moss criterion, as the photocurrent created by intrinsic absorption is sufficiently high. In all the obtained spectra of various samples, the value of $\Delta E_{g}$ coincides within the limits of an allowable error of calculation and equals at $300 \mathrm{~K} \Delta E_{g}=1.22 \pm 0.01 \mathrm{eV}$, and at $100 \mathrm{~K}$ $\Delta E_{g}=1.24 \pm 0.01 \mathrm{eV}$. At the quantum energy $1.30 \mathrm{eV}$ the photocurrent reaches its peak value and with increasing the energy monotonously decreases. The third characteristic area in the photoconductivity spectrum is the area close to $1.41 \mathrm{eV}$ where the photocurrent has some bend. With further increasing the quantum energy, the photocurrent decreases and in low-ohmic crystals it 


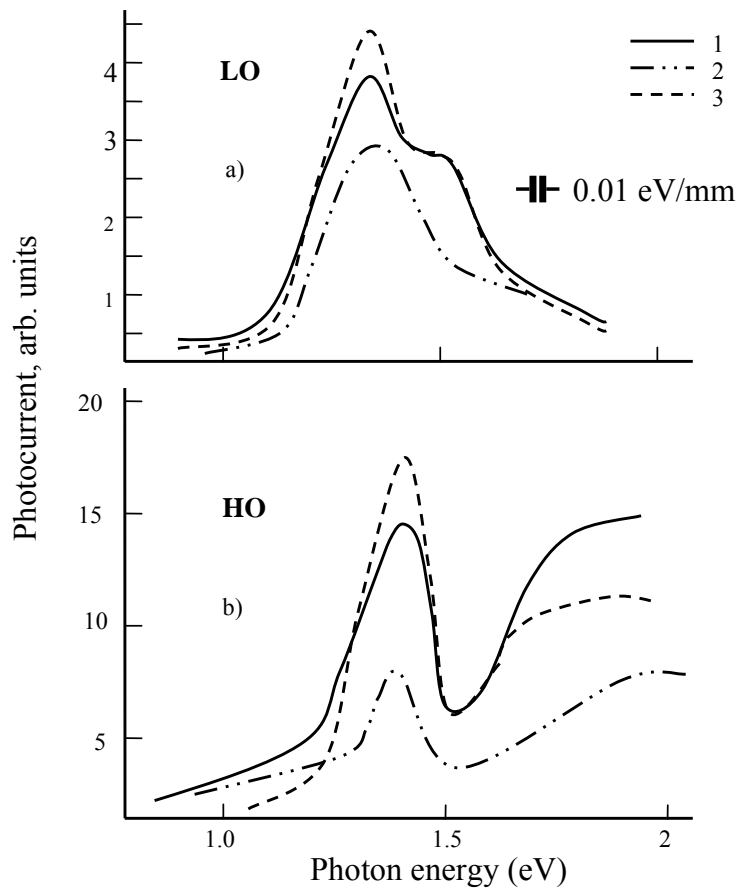

Fig. 1. Spectral dependence of the photocurrent for a lowresistance (a) and high-resistance (b) crystals of $\mathrm{Ag}_{3} \mathrm{In}_{5} \mathrm{Se}_{9}$ at $100 \mathrm{~K}$ (curves 1,3 ) and $300 \mathrm{~K}$ (curve 2). $\vec{E}$ light $\| \vec{c}$.

gradually tends to zero when reaching the quantum energy $2 \mathrm{eV}$. However, here high-ohmic crystals behave in another way. Since $1.5 \mathrm{eV}$ (the fourth area of a spectrum, Fig. 1b), the photocurrent grows and accepts the maximal value at $1.8 \mathrm{eV}$ at temperature $300 \mathrm{~K}$ and $1.87 \mathrm{eV}$ at $100 \mathrm{~K}$. As it is shown, this maximum is approximately $0.07 \mathrm{eV}$ shifted to the side of smaller energies with the growth of the sample temperature from 100 up to $300 \mathrm{~K}$.

It is necessary to note that the maximum or bends in the photoconductivity spectrum at the quantum energies exceeding $\Delta E_{g}$ are also found in single crystals of $\mathrm{AgInSe}_{2}$. In the work [2], it is explained with the fact that the spin-orbital splitting takes place in the valence band of $\mathrm{AgInSe}_{2}$, which is conditioned by the mixing the Ag $4 d$-levels with $p$-levels of other atoms. By this time, there formed three valence subbands. The lowest interband transitions from these $\mathrm{A}, \mathrm{B}$ and $\mathrm{C}$ subbands are equal $1.24,1.34$, and $1.6 \mathrm{eV}$, respectively. Probably, the same occurs in $\mathrm{Ag}_{3} \mathrm{In}_{5} \mathrm{Se}_{9}$ compounds. In comparison with $\mathrm{AgInSe}_{2}$, the relative number of $\mathrm{Ag}$ atoms in the formula unit of $\mathrm{Ag}_{3} \mathrm{In}_{5} \mathrm{Se}_{9}$ is less. In the photoconductivity spectrum of crystals $\mathrm{Ag}_{3} \mathrm{In}_{5} \mathrm{Se}_{9}$, photocurrent maxima corresponds to the quantum energies $1.30,1.41$, and $1.80 \mathrm{eV}$.

As seen, they strongly differ from the corresponding values of $\mathrm{AgInSe}_{2}$. In the work [2], it is also shown, that the photoconductivity spectrum shape strongly changes when turning the polarization vector of incident light around the normal to the surface of a sample. The amplitude of the found peaks depends on the light polarization. It is revealed by us that the spectrum of photoconductivity also depends on the direction of the applied electric field. Fig. 2 shows photoconductivity of high- and low-ohmic samples (curves 1 and 2, respectively) at $100 \mathrm{~K}$. The electric field $\vec{E}$ is directed along the axis $\vec{z}$ (in parallel to a crystal axis $\vec{c}$ ), and the light beam falls in parallel to axis $\vec{x}$. The spectrum differed from the spectra shown in Fig. 1, in range of quantum energies exceeding $1.5 \mathrm{eV}$. At $\vec{E} \| \vec{z}$ in the area above $1.5 \mathrm{eV}$ in the high-ohmic samples, the photocurrent has small increase, and then starting from $1.6 \mathrm{eV}$ remains constant. Some bend near $1.53 \mathrm{eV}$ is also inherent to some low-resistance samples. This characteristic part occupies a maximum in the interval $1.5 \ldots 1.95 \mathrm{eV}$ at $\vec{E} \perp \vec{z}$. Photosensivity of lowand high-ohmic samples in conditions $\vec{E} \| \vec{z}$ and $\vec{E}_{\text {light }} \perp \vec{x}$ in the specified interval of incident quantum energies when light is non-polarized can be accepted as a constant (independent from $h v$ ). It is necessary to note that all the abovementioned spectra of photoconductivity were measured inside the part of current-voltage characteristics (CVC) where the Ohm law is valid. The electric field strength is the same in all the parts of a spectrum and equals $10 \mathrm{~V} / \mathrm{cm}$. But CVC of high-ohmic samples obeys the Ohm law only up to $180 \mathrm{~V} / \mathrm{cm}$, the nonlinear part is further observed. For comparison in Fig. 3, we show the spectra of photoconductivity for

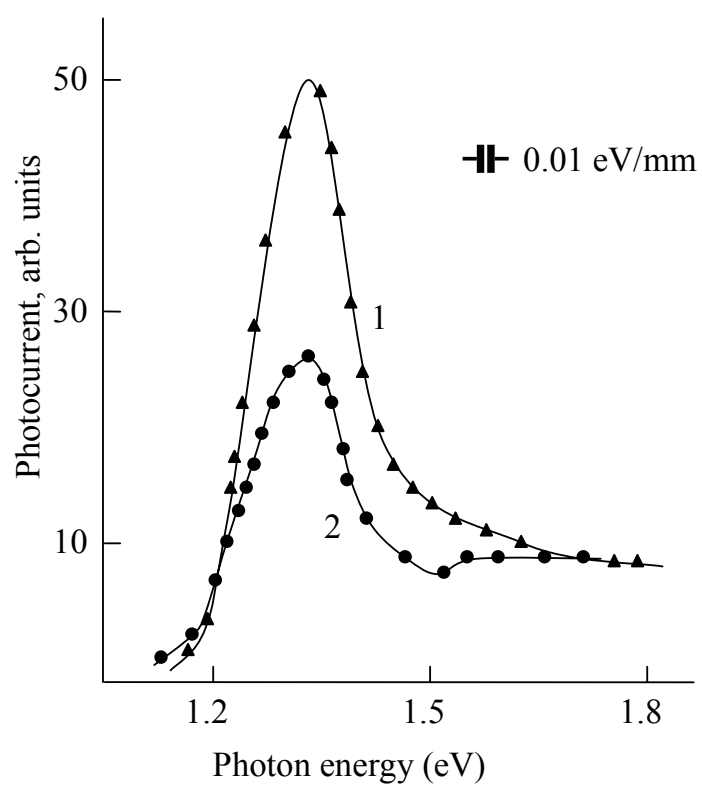

Fig. 2. Spectral dependence of the photocurrent for highresistance samples (curve 1) and low-resistance ones (curve 2)

$$
\rightarrow \quad \rightarrow \rightarrow \rightarrow
$$

of $\mathrm{Ag}_{3} \operatorname{In}_{5} \mathrm{Se}_{9}$ at $100 \mathrm{~K}$ and $\vec{E}$ light $\|\vec{x}, \vec{E}\| \vec{z}$. 
high-ohmic crystals at $100 \mathrm{~K}, \vec{E}_{\text {light }} \perp \vec{z}$, at strong ( $E=$ $350 \mathrm{~V} / \mathrm{cm}$, curve 1) and weak $(E=10 \mathrm{~V} / \mathrm{cm}$, curve 2) fields. Spectra were measured in the mode of constant illumination of samples, the intensity of illumination in both spectra was identical.

Photosensivity of the sample at $E=350 \mathrm{~V} / \mathrm{cm}$ begins with the energy of the incident quantum close to $0.95 \mathrm{eV}$. However, the spectrum 2 begins with the lower energy. Therefore, it is possible to tell that there is an electric decay of impurity photoconductivity. As it is clear from the figure, the photocurrent corresponding to the certain value of quantum, in the spectrum 1 is less than in the spectrum 2 within the interval $0.95 \ldots 1.7 \mathrm{eV}$. It is probably connected with reduction of the carrier mobility in strong electric fields (such a dependence takes place in dark electroconductivity). In the spectrum 1 , in the area around the first maximum of the photocurrent, there is a breakage. Here one can observe current instability, i.e., aperiodic oscillations of the photocurrent. The amplitude of the second photocurrent peak grows with the growth of the electric field, the peak is formed. It is possible to assume, that it is associated with the influence of strong electric field on defect surface states.

Shown in Fig. 4 are lux-ampere characteristics (LAC) of low- and high-ohmic samples in weak electric fields at $T=300 \mathrm{~K}$. The wavelength of incident light corresponded to the peak value of the photocurrent in the spectrum of photoconductivity. The straight line 1 expresses LAC of the high-ohmic samples. Presence and extent of various parts on LAC is usually determined by initial electron filling the $r$-centers [3]. With the growth of a filling degree the linear part of LAC gradually "supersedes" both the sublinear and saturation ones, as the $r$-centers are strongly exhausted as a result of recharging the centers already at rather weak excitation. Linear recombination observable in $\mathrm{Ag}_{3} \mathrm{In}_{5} \mathrm{Se}_{9}$ at $300 \mathrm{~K}$,

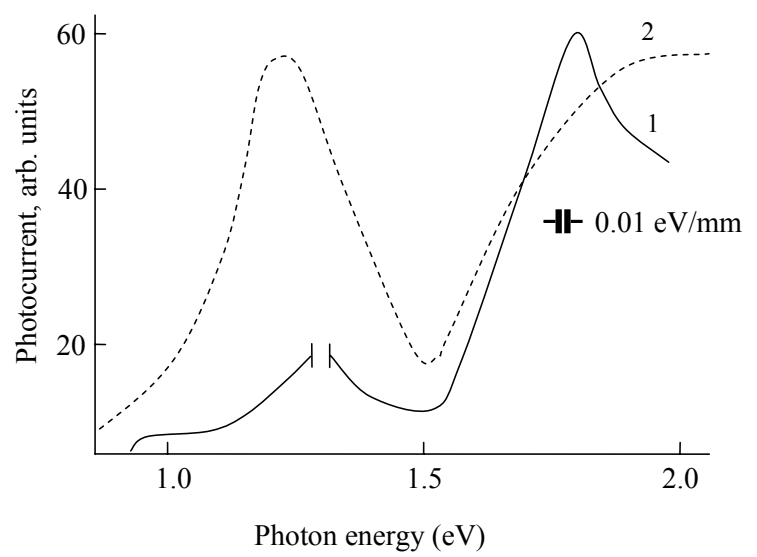

Fig. 3. Spectrum of the photocurrent of the high-resistance sample of $\mathrm{Ag}_{3} \mathrm{In}_{5} \mathrm{Se}_{9}$ at strong $(E=350 \mathrm{~V} / \mathrm{cm}$, curve 1) and weak $(E=10 \mathrm{~V} / \mathrm{cm}$, curve 2$)$ fields and $\vec{E}_{\text {light }} \perp \vec{z}, \vec{E} \| \vec{x}$. confirms that in the given interval of intensities ( $I$ ) electron and hole filling the $r$-centers remains practically constant, hence, $\tau_{n}$ does not depend on $I$. Curve 2 in the figure expresses LAC of low-resistance samples. It consists of two linear parts and a transitive sublinear part. Such course of LAC, probably, can be associated with capture of a hole with $r$-centers up to the small limited concentration corresponding to the inflection point.

The character of LAC for low- and high-ohmic samples at $100 \mathrm{~K}$ is identical. And LAC obeys the law of quadratic bimolecular recombination. It means that the mechanisms of recombination in both cases are the same.

Presence of a long-term relaxation of photoconductivity testifies to existence in crystals of levels of sticking, which were investigated by measurement of temperature dependence of thermo-stimulated current (TSC). The non-equilibrium carriers created at the preliminary excitation with the quantum of the energy $h v=1.22 \mathrm{eV}$, can be not captured only by repulsive centers. However, as it is known [4], the centers start to capture electrons at rather low $\vec{E}$ (about $10 \mathrm{~V} / \mathrm{cm}$ ). When this condition is satisfied in our experiments, levels of sticking have not been found. Therefore, at preliminary excitations it was necessary to increase the applied electric field gradually. Starting from $40 \mathrm{~V} / \mathrm{cm}$, the curve $\sigma(T)$ shows the TSC peak. With the further increase in the electric field $E_{\text {exc }}$, in the course of preliminary excitation, the level of filling the traps began to grow. The family of TSC curves at various fields of excitation is shown in Fig. 5. With increase of $E_{\text {exc }}$ up to

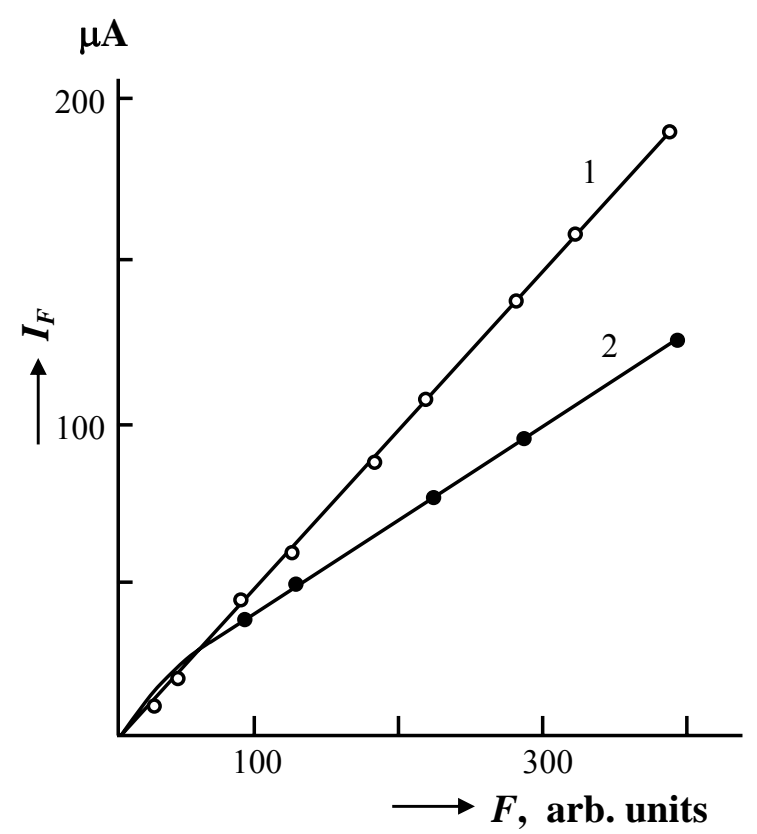

Fig. 4. Lux-ampere characteristics of the high-resistance (curve 1) and low-resistance (curve 2) $\mathrm{Ag}_{3} \mathrm{In}_{5} \mathrm{Se}_{9}$ at $300 \mathrm{~K}$. 


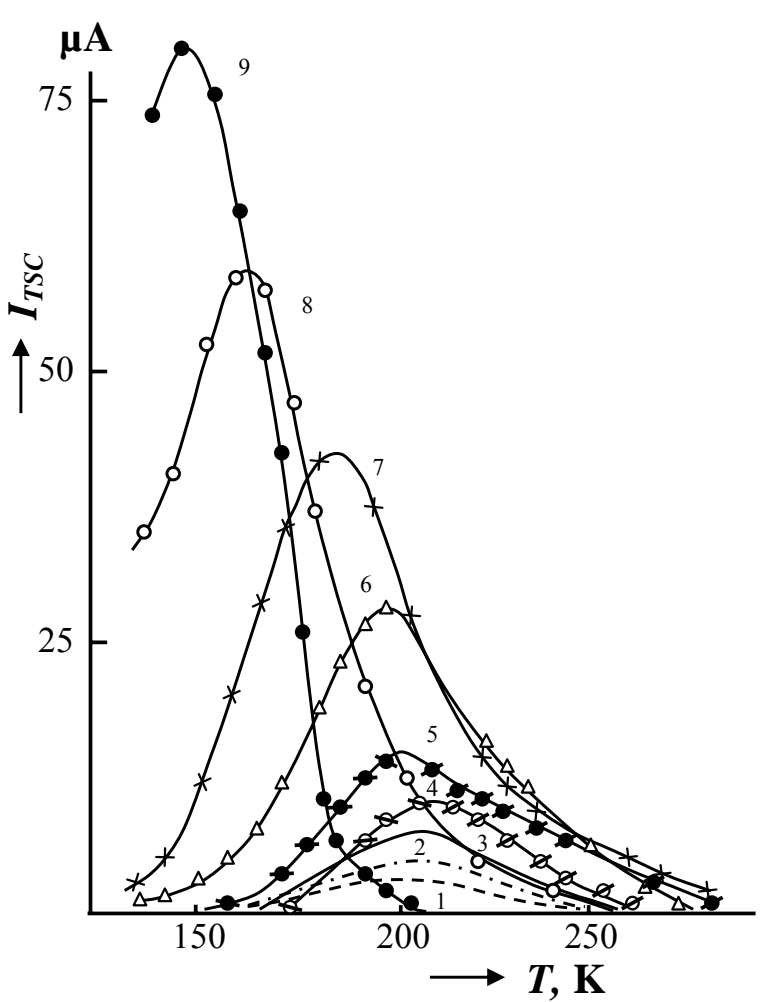

Fig. 5. TSC curve for $\mathrm{Ag}_{3} \mathrm{In}_{5} \mathrm{Se}_{9}$ at various fields after preliminary excitation; $E$ equals: $40 \mathrm{~V} / \mathrm{cm}(1), 70(2), 120(3)$, 180 (4), 240 (5), 320 (6), 500 (7), $700(8), 1000$ (9).

$180 \mathrm{~V} / \mathrm{cm}$, the temperature $\left(T_{m}\right)$ corresponding to maximum TSC grows, the further increase of $E_{\text {exc }}$ up to $350 \mathrm{~V} / \mathrm{cm}$ practically does not change $T_{m}$, but above $350 \mathrm{~V} / \mathrm{cm}$ with the growing $E_{\text {exc }}$, on the contrary, $T_{m}$ decreases. Similar influence of an electric field on levels of sticking was revealed in the work [5]. Authors concluded that with the growth of the voltage, the TSC peak corresponds to a deeper Fermi quasi-level for traps. The same occurs, probably, in this case. Depth of deposition of a level of the sticking, found of the analysis of TSC curves, is equal to $E_{t}=0.12 \mathrm{eV}$.
With the purpose to ascertain the mechanism of TSC recombination, we investigated the dependence of $T_{m}$ on time of excitation at the fixed values of $E_{\text {exc }}$. It appeared that $T_{m}$ does not depend on the excitation time. Hence, the found level of sticking is slow.

For the maximal value of the capture cross-section, we obtained $S=2.5 \cdot 10^{-19} \mathrm{~cm}^{2}$. Thus, the level of sticking observed by us is repulsive.

The research of the absorption spectra at various temperatures has shown that the edge of intrinsic absorption is formed by direct interband transitions. The obtained values of the forbidden gap, its temperature coefficient completely coincide with the results of photoelectric researches.

\section{References}

1. V.I. Tahirov, N.F. Gahramanov, H.A. Huseynov, F.M. Aliev, G.G. Huseynov, New class of ternary semiconductor compounds of $\mathrm{A}_{3}^{\mathrm{I}} \mathrm{B}_{5}^{\mathrm{III}} \mathrm{C}_{9}^{\mathrm{VI}}$ type // Kristallografiya 25, No 2, p. 411-413 (1980) (in Russian).

2. V.I. Tahirov, H.A. Huseynov, M.B. Djafarov, Stimulation of low-frequency oscillation of current in $\mathrm{Ag}_{3} \mathrm{In}_{5} \mathrm{Se}_{9} / /$ Tekhnich. Fizika 60, No 10, p. 190-192 (1990) (in Russian).

3. V.I. Tahirov, N.F. Gahramanov, H.A. Huseynov, New class of ternary semiconductor compounds of $\mathrm{A}_{3}^{\mathrm{I}} \mathrm{B}_{5}^{\mathrm{III}} \mathrm{C}_{9}^{\mathrm{VI}}$ type. Baku State University, Baku, 2001, p. 303.

4. V.E. Lashkaryov, A.V. Lyubchenko, M.K. Sheynkman, Non-equilibrium processes in photoconductors. Naukova dumka, Kiev, 1981, p. 248 (in Russian).

5. N.G. Jdanov, M.S. Kagan, S.G. Kalashnikov, Recombination of hot electrons by repulsive impurity centers in hermanite // Fizika tverdogo tela 8, No 3, p. 774-782 (1966) (in Russian).

6. L.Ab. Dusell, R.H. Buba // J. Appl. Phys. 37, No 7, p. 2797-2807 (1966).

7. B. Tell, E.M. Hamonds, P.M. Bridenbangh, H.M. Kasper // J. Appl. Phys. 46, p. 2998-3001 (1975). 